\title{
New Results on Start-Points for Multi-Valued Maps
}

\author{
Yaé Ulrich Gaba ${ }^{1,2}$, Erdal Karapınar ${ }^{3,4,5, *}$, , Adrian Petruşel ${ }^{6,7}\left(\mathbb{D}\right.$ and Stojan Radenović ${ }^{8}$ \\ 1 Institut de Mathématiques et de Sciences Physiques (IMSP), Porto-Novo 01 BP 613, Benin; \\ yaeulrich.gaba@gmail.com \\ 2 African Center for Advanced Studies (ACAS), P.O. Box 4477, Yaoundé, Cameroon \\ 3 Division of Applied Mathematics, Thu Dau Mot University, Thu Dau Mot City 820000, \\ Binh Duong Province, Vietnam \\ 4 Department of Mathematics, Çankaya University, Etimesgut, Ankara 06790, Turkey \\ 5 Department of Medical Research, China Medical University Hospital, China Medical University, \\ Taichung 40402, Taiwan \\ 6 Faculty of Mathematics and Computer Science, Babeş-Bolyai University Cluj-Napoca, Str.Mihail \\ Kogălniceanu 1, 400000 Cluj-Napoca, Romania; petrusel@math.ubbcluj.ro \\ 7 Academy of Romanian Scientists, Str. Splaiul Independenţei 54, Sector 5, RO-050094 Bucharest, Romania \\ 8 Faculty of Mechanical Engineering, University of Belgrade, Kraljice Marije 16, 11120 Beograd, Serbia; \\ radens@beotel.rs \\ * Correspondence: erdalkarapinar@tdmu.edu.vn or erdalkarapinar@yahoo.com
}

Received: 3 November 2020; Accepted: 28 November 2020; Published: 3 December 2020

\begin{abstract}
In this manuscript we investigate the existence of start-points for the generalized weakly contractive multi-valued mappings in the setting of left $K$-complete quasi-pseudo metric space. We provide an example to support the given result.
\end{abstract}

Keywords: quasi-pseudometric; start-point; end-point; fixed point; weakly contractive

MSC: Primary 47H05; Secondary 47H09; 47H10; 54H25

\section{Introduction and Preliminaries}

The quasi-pseudo metric space, which is obtained by relaxing the symmetry condition, is one of the refinements of the notion of metric space. In the point view of fixed point theory, the lack of the symmetry axiom leads to consider the orientation in this new structure. Roughly speaking, fixed points for mappings are usually limits of the Picard sequence, which is constructed by the recursive iteration of the operator by starting with an arbitrarily chosen point. On the other hand, in this new structure, the distance function is not symmetric. Consequently, for an arbitrary initial value $\xi_{0}$, the value of the distance from its $n$-th iteration, $T^{n} x_{0}$, to its limit, say $x^{*}$ (if exists), and the value of the distance from its limit, $x^{*}$ (if exists), to its $n$-th iteration, $T^{n} x_{0}$, need not be equal. Under this motivation, the notions of start-point, end-point, $\varepsilon$-start-point, and $\varepsilon$-end-point were defined in [1]. In other words, fixed point has been investigated in the oriented structure, quasi-pseudo metric space, under the names of start-point and end-point. It is clear that, under the condition symmetry, the start-points and end-points coincide with the fixed points [2-5].

An initial result in the theory of start-point was given in [1] in order to extend the idea of fixed points for multi-valued mappings defined on quasi-pseudo metric spaces. A series of three papers, see $[1,6,7]$, has given a more or less detailed introduction to the subject. The theory of start-point came to extend the idea of fixed points for multi-valued mappings that are defined on quasi-pseudo metric spaces. More detailed introduction to the subject can be read in [1,6-13]. 
In this paper, we investigate the existence of start-points and end-points for a class of mappings, which are known as generalized weakly contractive multi-valued maps, in the context of left K-complete quasi-pseudo metric space.

Intuitively, as we mentioned above, the appropriate framework for the theory of start-point is the quasi-metric setting. For the sake of completeness, we recollect, in the present manuscript, the necessary notations and fundamental concepts from the literature. We first recall the basic notions regarding quasi-metric spaces as well as some additional definitions that are related to multi-valued maps on these spaces[14-16]. For a general approach in metric fixed point theory for multi-valued operators, see [17-19].

Definition 1 (See [1]). Let $q: X \times X \rightarrow[0, \infty)$ be a function where $X$ is a non-empty set. The function is called a quasi-pseudometric (respectively, $T_{0}$-quasi-metric) on $X$ if $\left(q_{1}\right)$ and $\left(q_{2}\right)$ (respectively, $\left(q_{1}\right)^{*}$ and $\left(q_{2}\right)$ ) hold, where

$\left(q_{1}\right) q(\xi, \xi)=0 \quad$ for all $\xi \in X$

$\left(q_{1}\right)^{*} q(\xi, \eta)=0=q(\eta, \xi)$ implies $\xi=y$, and

$\left(q_{2}\right) \quad q(\xi, \zeta) \leq q(\xi, \eta)+q(\eta, \zeta) \quad$ for all $\xi, \eta, \zeta \in X$

Note that the condition $\left(q_{1}\right)^{*}$ is known as the $T_{0}$-condition. Furthermore, for a quasi-pseudo metric $q$ on $X$, the function $q^{-1}: X \times X \rightarrow[0, \infty)$, which is defined by $q^{-1}(\xi, \eta)=q(\eta, \xi)$ for all $\xi, \eta \in X$, forms a quasi-pseudo metric on the same set $X$ and is named as the conjugate of $q$. For a $T_{0}$-quasi-metric $d$ on $X$, a distance function $d_{q}: X \times X \rightarrow[0, \infty)$, defined by $d_{q}(\xi, \eta)=\max \{q(\xi, \eta), q(\eta, \xi)\}$ for all $(\xi, \eta) \in X \times X$, becomes a metric on $X$.

Remark 1. In some sources, the quasi-pseudo metric is called hemi-metric (see [20]). Moreover, $T_{0}$-quasi-metric is known also as a quasi-metric in the literature.

In what follows, we consider three well-known examples in order to illustrate the validity of Definition 1.

Example 1 (Truncated difference). Set $\mathbb{R}_{0}^{+}:=[0, \infty)$ and $\delta: \mathbb{R}_{0}^{+} \times \mathbb{R}_{0}^{+} \rightarrow \mathbb{R}_{0}^{+}$be given, for any $\xi, \eta \in X$, by

$$
\delta(\xi, \eta)=\max \{0, \xi-\eta\}
$$

Under these conditions, $\delta$ forms a $T_{0}$-quasi-metric. Further, the pair $\left(\mathbb{R}_{0}^{+}, \delta\right)$ becomes a $T_{0}$-quasi-metric space.

Example 2 (cf. [21]). Let $A, B$ be two non-empty set, such that $A \cap B \neq \varnothing$. Set $X=A \cup B$ and $q: X \times X \rightarrow[0, \infty)$ be given, for any $a, b \in X$, by

$$
q(a, b)=\left\{\begin{array}{rr}
0 & \text { if } a=b \\
\frac{3}{2} & \text { if } a \in A, b \in B \\
2 & \text { if } b \in A, a \in B \\
1 & \text { otherwise }
\end{array}\right.
$$

Under these conditions, $q$ forms a $T_{0}$-quasi-metric. Further, the pair $(X, q)$ becomes a $T_{0}$-quasi-metric space.

Example 3 (cf. [22]). Set $\mathbb{I}:=[0,1]$, and define $\delta: \mathbb{I} \times \mathbb{I} \rightarrow \mathbb{R}_{0}^{+}$be defined as

$$
\delta(\xi, \eta)= \begin{cases}\xi-\eta, & : \xi \geq \eta, \\ 1, & : \xi<\eta .\end{cases}
$$

Under these conditions, $\delta$ forms a quasi-pseudo metric that is obviously not $T_{0}$. 
For a quasi-pseudo metric space $(X, q)$, we define an open $\varepsilon$-ball at a point $\xi$ as follows: For $\xi \in X$ and $\varepsilon>0$,

$$
B_{q}(\xi, \varepsilon)=\{\eta \in X: q(\xi, \eta)<\varepsilon\}
$$

Let $(X, q)$ be a quasi-pseudo metric space. We say that the sequence $\left\{\xi_{n}\right\}$ is $q$-convergent to $\xi$ (or left-convergent to $\xi)$, if

$$
q\left(\xi_{n}, \xi\right) \longrightarrow 0
$$

and we denote this fact by $\xi_{n} \stackrel{q}{\longrightarrow} \xi$. More precisely, $\left\{\xi_{n}\right\}$ converges to $\xi$ with respect to $\tau(q)$.

In a similar manner, a sequence $\left\{\xi_{n}\right\}$ is $q^{-1}$-convergent to $\xi$ (or right-convergent to $\xi$ ), if

$$
q\left(\xi, \xi_{n}\right) \longrightarrow 0
$$

fact denoted by $\xi_{n} \stackrel{q^{-1}}{\longrightarrow} \xi$. Actually, $\left\{\xi_{n}\right\}$ converges to $\xi$ with respect to $\tau\left(q^{-1}\right)$

A sequence $\left\{\xi_{n}\right\}$, in the setting of a quasi-pseudo metric space $(X, q)$, is said to be $d_{q}$-convergent to $\xi$ in the case the sequence converges to $\xi$ from left and right, which is,

$$
\xi_{n} \stackrel{q}{\longrightarrow} \xi \text { and } \xi_{n} \stackrel{q^{-1}}{\longrightarrow} \xi
$$

Moreover, it is denoted as $\xi_{n} \stackrel{d_{q}}{\longrightarrow} \xi$ (or, $\xi_{n} \longrightarrow \xi$, if there is no confusion).

Remark 2. From the definition of $d_{q}$-convergence, we have

$$
d_{q} \text {-convergence implies } q \text {-convergence. }
$$

The reverse implication does not hold in general, as demonstrated in the following example.

Example 4 (cf. [22]). Set $\mathbb{I}:=[0,1]$, and define $q: \mathbb{I} \times \mathbb{I} \rightarrow \mathbb{R}_{0}^{+}$be defined as

$$
q(\xi, \eta)= \begin{cases}0 & : \xi \leq y \\ 1 & : \xi>y\end{cases}
$$

Subsequently, it is evident that $(X, q)$ forms a quasi-pseudo metric space.

Consider

$$
\xi_{n}=\left\{\begin{array}{lc}
\frac{1}{2}+2^{-n} & : n \text { is odd } \\
\frac{1}{3}+3^{-n} & : n \text { is even }
\end{array}\right.
$$

It is easy to see that the sequence $\left\{\xi_{n}\right\}$ is right-convergent (to $1 / 3$ ) and left-convergent (to 1 ), but not $d_{q}$-convergent.

Definition 2 (See e.g., [1]). A sequence $\left\{\xi_{n}\right\}$ in a quasi-pseudo metric space $(X, q)$ is called left K-Cauchy if for every $\varepsilon>0$, there exists $n_{0} \in \mathbb{N}$, such that

$$
\text { for all } n, k: n_{0} \leq k \leq n \quad q\left(\xi_{k}, \xi_{n}\right)<\varepsilon \text {; }
$$

Similarly, we define right $K$-Cauchy sequences and observe that a sequence is left $K$-Cauchy with respect to $q$ if and only if it is right $K$-Cauchy with respect to $q^{-1}$.

Example 5 (See [8]). Set $\mathbb{I}:=(0,1)$, and define $\delta: \mathbb{O} \times \mathbb{O} \rightarrow \mathbb{R}_{0}^{+}$be defined as

$$
q(\xi, \eta)= \begin{cases}\xi-\eta & : \xi \geq \eta \\ 1 & : \xi<\eta\end{cases}
$$


Let us define the sequence $\left\{\xi_{n}\right\}$ given by $\xi_{n}=(n+1)^{-1}$. Subsequently,

$$
q\left(\xi_{r}, \xi_{s}\right)<r^{-1}
$$

for all $s>r$; hence, $\left\{\xi_{n}\right\}$ is left K-Cauchy. However, $\left\{\xi_{n}\right\}$ is not right K-Cauchy, since whenever $\xi \in X, q\left(\xi_{m}, \xi\right)=1$ after a certain stage. On the other hand, if one considers the sequence $\left\{\eta_{n}\right\}$ where $\eta_{n}=1-(n+1)^{-1}$, one could easily see that it is right K-Cauchy.

Definition 3 (See $[1,13])$. We say that $(X, q)$ is left-K-complete if any left K-Cauchy sequence is q-convergent. Furthermore, we say that quasi-pseudo metric space $(X, q)$ is Smyth complete if any left K-Cauchy sequence is $d_{q}$-convergent.

It is easy to see that every Smyth-complete quasi-metric space is left K-complete [13], and the converse implication does not hold.

Definition 4 ([1]). We say that a $T_{0}$-quasi-metric space $(X, q)$ is said to be bicomplete if the corresponding metric $d_{q}$ on $X$ is complete.

Example 6. Let us again consider Example 1. In that case, for any $\xi, \eta \in X=[0, \infty)$, we have that $d_{q}(\xi, \eta)=\max \{\xi-\eta, \eta-\xi\}=|\xi-\eta|$. We know that $(\mathbb{R},||$.$) is a complete metric space; hence, ([0, \infty),||$. is an example of bicomplete $T_{0}$-quasi-metric space.

However, if we take the quasi-pseudo metric that is defined in Example 3, it is clear that $(X, \delta)$ is not bicomplete, since $(X, \delta)$ is not even $T_{0}$.

Definition 5 ([1]). Let $A$ be a subset of a quasi-pseudo metric space $(X, q)$. We say that $A$ is bounded if there exists a $\Delta>0$, such that $q(\xi, \eta)<\Delta$ whenever $\xi, \eta \in A$.

\section{Example 7.}

1. Let $X=\{a, b, c\}$. The map $q: X \times X \rightarrow[0, \infty)$ defined by $q(a, b)=q(a, c)=0, q(b, a)=q(b, c)=1$, $q(c, a)=q(c, b)=2$ and $q(\xi, \xi)=0$ for all $\xi \in X$ is a bounded $T_{0}$-quasi-metric on $X$. Indeed, for any $\xi, \eta \in X, q(\xi, \eta) \leq 2$.

2. The quasi-pseudo metric presented in Example 4 is bounded, as for any $\xi, \eta \in X, q(\xi, \eta) \leq 1$.

Let $(X, q)$ be a quasi-pseudo metric space. We set $\mathscr{P}_{0}(X):=\mathscr{P}_{0}(X) \backslash\{\varnothing\}$, where $\mathscr{P}_{0}(X)$ denotes the power set of $X$.

$$
\begin{aligned}
\mathscr{P}_{c b}(X): & =\left\{A \in \mathscr{P}_{0}(X): A \text { closed and bounded }\right\} \\
\mathscr{P}_{k}(X): & =\left\{A \in \mathscr{P}_{0}(X): A \text { compact }\right\} \\
\mathscr{P}_{c}(X): & =\left\{A \in \mathscr{P}_{0}(X): A \text { closed }\right\} .
\end{aligned}
$$

For $\xi \in X$ and $A \in \mathscr{P}_{0}(X)$, we set:

$$
q(\xi, A):=\inf \{q(\xi, a), a \in A\}, \quad q(A, \xi):=\inf \{q(a, \xi), a \in A\} .
$$

We also define the map $H: \mathscr{P}_{0}(X) \times \mathscr{P}_{0}(X) \rightarrow[0, \infty]$ by

$$
H(A, B)=\max \left\{\sup _{a \in A} q(a, B), \sup _{b \in B} q(A, b)\right\} \text { whenever } A, B \in \mathscr{P}_{0}(X) .
$$

Subsequently, the distance function $H$ is called the Hausdorff extended quasi-pseudo metric on $\mathscr{P}_{0}(X)$. Notice that, here, the word "extended" is use to emphasize that $H$ can attain the value $\infty$ as it appears in the definition. 
Finally, we recall some concepts that are related to the classical fixed point notions in the setting of a quasi-pseudo metric space.

Definition 6 (cf.[1]). Let $(X, q)$ be a quasi-pseudo metric space and $F: X \rightarrow \mathscr{P}_{0}(X)$ be a multi-valued map. Suppose that $H$ is a Hausdorff quasi-pseudo metric on $\mathscr{P}_{0}(X)$. We say that $\xi \in X$ is

(i) a fixed point of $F$ if $\xi \in F \xi$,

(ii) a strict fixed point if $F \xi=\{\xi\}$,

(iii) a start-point of $F$ if $H(\{\xi\}, F \xi)=0$, and

(iv) an end-point of $F$ if $H(F \xi,\{\xi\})=0$.

In this context, we can also write $H(\eta, F \eta):=H(\{\eta\}, F \eta), \eta \in X$. Notice that $H(\{\eta\}, F \eta)=$ $\sup _{\psi \in F \eta} q(\eta, \psi)$, while $H(F \eta,\{\eta\})=\sup _{\psi \in F \eta} q(\psi, \eta)$.

\section{Main Results}

In this section, we give a new start-point theorem for a generalized weakly contractive multi-valued map.

As we dive into the topic, it could be very interesting to point out this known fact, which is always good to remember. That is, if $\xi$ is both a start-point and an end-point of a multi-valued $F$, then $\xi$ is a fixed point of $F$. In fact, $F \xi$ is a singleton. Observe that a fixed point of a multi-valued $F$ need not be a start-point or an end-point. We provide the following three examples in order to illustrate that fact.

Example 8. Consider the $T_{0}$-quasi-pseudo metric space $(X, q)$, where $X=\{a, b, c\}$ and $q$ defined by $q(a, b)=q(a, c)=0, q(b, a)=q(b, c)=2, q(c, a)=q(c, b)=4$ and $q(\xi, \xi)=0$ for $\xi=a, b, c$. The multi-valued map $F: X \rightarrow \mathscr{P}_{0}(X)$ is considered by $F a=\{a, b\}$ and $F \xi=X \backslash\{\xi\}$ for $\xi=b, c$. Obviously, $a$ is a fixed point for $F$. Moreover, since

$$
H(\{a\}, F a)=\max \{q(a, a), q(a, b)\}=0,
$$

we derive that $a$ is a start-point, but, since

$$
H(F a,\{a\})=\max \{q(a, a), q(b, a)\}=2 \neq 0,
$$

we derive that $a$ is not an end-point. Furthermore, there is no other start-point or end-point for F.

Example 9. Consider the $T_{0}$-quasi-pseudo metric space $(X, q)$, as defined in the previous example (Example 8). The multi-valued map $F: X \rightarrow \mathscr{P}_{0}(X)$ is considered by $F \xi=\{a, b\}$ for $\xi=a, b, c$. Obviously, $a, b$ are fixed points for F. Again, a is a start-point, but not an end-point. Observe this time around that $b$ is an end-point, but not a start-point.

Example 10. Consider the $T_{0}$-quasi-pseudo metric space $(X, q)$, as defined in the previous example (Example 8). The multi-valued map $F: X \rightarrow \mathscr{P}_{0}(X)$ is considered by $F a=\{b\}, F b=\{c\}, F c=\{a\}$. The map $F$ does not have any fixed point. However, we can easily that $a$ is the only start-point and $c$ the only end-point for $F$.

Remark 3. So far in the examples, we have been obtaining fixed points. Let us observe what happens when we are in the presence of a strict fixed point.

Example 11. Consider the $T_{0}$-quasi-pseudo metric space $(X, q)$, where $X=\{a, b, c\}$ and $q$ defined by $q(a, b)=$ $q(a, c)=q(b, c)=0, q(b, a)=2, q(c, a)=q(c, b)=4$ and $q(\xi, \xi)=0$ for $\xi=a, b, c$. We define, on $X$, the multi-valued map $F: X \rightarrow \mathscr{P}_{0}(X)$ by $F a=\{a\}$ and $F b=F c=\{b, c\}$ for $\xi=b, c$.

$$
H(\{a\}, F a)=q(a, a)=0,
$$


and

$$
H(F a,\{a\})=q(a, a)=0,
$$

i.e., $a$ is is both a start-point and an end-point for $F$.

The point $b$ is both a fixed point (which is not strict) and end-point for $F$, while $c$ is neither a (strict) fixed point nor a start-point nor an end-point for $F$.

In fact, the above example illustrates the following fact:

Lemma 1. Let $X$ be non-empty set and $H$ the Hausdorff quasi-pseudo metric that is derived by a quasi-pseudo metric q. Let $F: X \rightarrow \mathscr{P}_{0}(X)$ be a multi-valued map. If $\xi \in X$ is a strict fixed point, then $\xi$ is both a start-point and an end-point.

Proof. The result is immediate, since, for $F \xi=\{\xi\}$, we have

$$
H(\{\xi\}, F \xi)=q(\xi, \xi)=0=q(\xi, \xi)=H(F \xi,\{\xi\})=0 .
$$

We begin with the following intermediate result.

Lemma 2. Let $(X, q)$ be $T_{0}$-quasi-metric space and $A \subset X$. If $A$ is a compact subset of $\left(X, d_{q}\right)$, then it is a closed subset of $(X, q)$. That is, $\mathscr{P}_{k}(X) \subset \mathscr{P}_{c}(X)$.

Proof. Let $\left\{\xi_{n}\right\}$ be a sequence in $A$, such that $q\left(\xi_{,} \xi_{n}\right) \rightarrow 0$ for some $\xi \in X$. Because $A$ is a compact subset of $\left(X, d_{q}\right)$, there exists a subsequence $\left\{\xi_{n_{k}}\right\}$ of $\left\{\xi_{n}\right\}$ and a point $\zeta \in A$, such that $d_{q}\left(\zeta, \xi_{n_{k}}\right) \rightarrow 0$. Thus, we have $q\left(\xi_{n_{k}}, \zeta\right) \rightarrow 0$. While using the triangle inequality, we have

$$
q(\xi, \zeta) \leq q\left(\xi, \xi_{n_{k}}\right)+q\left(\xi_{n_{k}}, \zeta\right)
$$

Letting $k \rightarrow \infty$ in above inequality, we obtain $\xi=\zeta$ and $\xi \in A$. Thus, $A$ is a closed subset of $(X, q)$.

The concept of weakly contractive maps that appeared in [23] (Definition 1) is one of the generalizations of contractions on metric spaces. In [23], the authors defined such maps for single valued maps on Hilbert spaces and proved the existence of fixed points. Later, it was shown that most of the results of [23] still hold in any Banach space, see e.g., Rhoades[24-29]. As it is expected, this notion was extended to multi-valued maps and it was characterized in the setting of quasi-metric spaces.

In the literature, one of the useful auxiliary function is the comparison function that is initiated by [30], and, later, discussed and investigated densely by Rus [31] and many others. A function $\varphi:[0, \infty) \rightarrow[0, \infty)$ is called a comparison function [30,31] if it is increasing and $\varphi^{n}(t) \rightarrow 0$ as $n \rightarrow \infty$ for every $t \in[0, \infty)$, where $\varphi^{n}$ is the $n$-th iterate of $\varphi$. A simple example of such mappings is $\varphi(t)=\frac{k t}{n}$, where $k \in[0,1)$ and $n \in\{2,3, \cdots\}$.

Let $\Gamma$ be the family of functions $\gamma:[0, \infty) \rightarrow[0, \infty)$ satisfying the following conditions:

$\left(\Gamma_{1}\right) \gamma$ is nondecreasing;

$\left(\Gamma_{2}\right) \sum_{n=1}^{+\infty} \gamma^{n}(t)<\infty$ for all $t>0$.

Subsequently, a function $\phi \in \Gamma$ is called (c)-comparison function, see also [31,32].

Lemma 3 ([31]). If $\gamma:[0, \infty) \rightarrow[0, \infty)$ is a comparison function, then

1. each iterate $\gamma^{k}$ of $\gamma, k \geq 1$ is also a comparison function;

2. $\gamma$ is continuous at 0 ; and, 
3. $\gamma(t)<t$ for all $t>0$.

The listed properties above are also valid for (c)-comparison functions, since the class of (c)-comparison functions is a subclass of comparison functions.

For our own purpose, we introduce the $(c)^{*}$-comparison function, as follows:

Definition 7. A function $\gamma:[0, \infty) \rightarrow[0, \infty)$ is called $a(c)^{*}$-comparison function if

$\left(\gamma_{1}\right) \quad \gamma$ is nondecreasing with $\gamma(0)=0$ and $0<\gamma(t)<t$ for each $t>0$; and,

$\left(\gamma_{2}^{*}\right)$ for any sequence $\left\{t_{n}\right\}$ of $(0, \infty), \sum_{n=1}^{\infty} \gamma\left(t_{n}\right)<\infty$ implies $\sum_{n=1}^{\infty} t_{n}<\infty$.

Definition 8. Let $(X, q)$ be $T_{0}$-quasi-metric space.

1. A multi-valued map $F: X \rightarrow \mathscr{P}_{0}(X)$ is called weakly contractive if there exists a $(c)^{*}$-comparison function $\gamma$, such that, for each $\xi \in X$ there exists $\eta \in F \xi$ satisfying

$$
H(\eta, F \eta) \leq q(\xi, \eta)-\gamma(q(\xi, \eta)) .
$$

2. A single-valued map $f: X \rightarrow X$ is called weakly contractive if there exists $a(c)^{*}$-comparison function $\gamma$, such that

$$
q(f \xi, f \eta) \leq q(\xi, \eta)-\gamma(q(\xi, \eta)), \text { for every } \xi, \eta \in X
$$

The following is the main result of the paper.

Theorem 1. Let $(X, q)$ be a left K-complete quasi-pseudo metric space, $F: X \rightarrow \mathscr{P}_{c b}(X)$ be a weakly contractive multi-valued mapping. Subsequently, F has a start-point in X.

Proof. Let $\xi_{0} \in X$ be arbitrary. By (2), there exists $\xi_{1} \in F \xi_{0}$, such that, for every $\xi_{2} \in F \xi_{1}$, we have

$$
q\left(\xi_{1}, \xi_{2}\right) \leq H\left(\xi_{1}, F \xi_{1}\right) \leq q\left(\xi_{0}, \xi_{1}\right)-\gamma\left(q\left(\xi_{0}, \xi_{1}\right)\right) .
$$

Again, by (2), there exists an element $\xi_{2} \in F \xi_{1}$, such that, for every $\xi_{3} \in F \xi_{2}$, we have

$$
q\left(\xi_{2}, \xi_{3}\right) \leq H\left(\xi_{2}, F \xi_{2}\right) \leq q\left(\xi_{1}, \xi_{2}\right)-\gamma\left(q\left(\xi_{1}, \xi_{2}\right)\right) \leq q\left(\xi_{1}, \xi_{2}\right) \leq H\left(\xi_{1}, F \xi_{1}\right) .
$$
we have

Continuing this process, we can find a sequence $\left\{\xi_{n}\right\} \subset X$, such that, for $n \in\{0,1,2, \cdots\}$,

$$
\xi_{n+1} \in F \xi_{n}
$$

and

$$
q\left(\xi_{n+1}, \xi_{n+2}\right) \leq H\left(\xi_{n+1}, F \xi_{n+1}\right) \leq q\left(\xi_{n}, \xi_{n+1}\right)-\gamma\left(q\left(\xi_{n}, \xi_{n+1}\right)\right) \leq q\left(\xi_{n}, \xi_{n+1}\right) \leq H\left(\xi_{n}, F \xi_{n}\right) .
$$

Thus, the sequence $\left\{q\left(\xi_{n}, \xi_{n+1}\right)\right\}$ is non-increasing and so we can conclude that $\lim _{n \rightarrow \infty} q\left(\xi_{n}, \xi_{n+1}\right)=l$ for some $l \geq 0$. We show that $l=0$. Suppose that $l>0$. Subsequently, we have

$$
q\left(\xi_{n}, \xi_{n+1}\right) \leq q\left(\xi_{n-1}, \xi_{n}\right)-\gamma\left(q\left(\xi_{n-1}, \xi_{n}\right)\right) \leq q\left(\xi_{n-1}, \xi_{n}\right)-\gamma(l),
$$

and so

$$
q\left(\xi_{n+N}, \xi_{n+N+1}\right) \leq q\left(\xi_{n-1}, \xi_{n}\right)-N \gamma(l),
$$

which is a contradiction for $N$ large enough. Thus, we have

$$
\lim _{n \rightarrow \infty} q\left(\xi_{n}, \xi_{n+1}\right)=0
$$


For $m \in \mathbb{N}$ with $m \geq 3$, we have

$$
\begin{aligned}
q\left(\xi_{m-1}, \xi_{m}\right) & \leq q\left(\xi_{m-2}, \xi_{m-1}\right)-\gamma\left(q\left(\xi_{m-2}, \xi_{m-1}\right)\right) \cdots \\
& \leq q\left(\xi_{1}, \xi_{2}\right)-\gamma\left(q\left(\xi_{1}, \xi_{2}\right)\right)-\cdots-\gamma\left(q\left(\xi_{m-2}, \xi_{m-1}\right)\right)
\end{aligned}
$$

Hence, we get

$$
\sum_{k=1}^{m-2} \gamma\left(q\left(\xi_{k}, \xi_{k+1}\right)\right) \leq q\left(\xi_{1}, \xi_{2}\right)-q\left(\xi_{m-1}, \xi_{m}\right)
$$

Letting $m \rightarrow \infty$ in above inequality, we obtain

$$
\sum_{k=1}^{\infty} \gamma\left(q\left(\xi_{k}, \xi_{k+1}\right)\right) \leq q\left(\xi_{1}, \xi_{2}\right)<\infty
$$

which implies, using $\left(\gamma_{2}^{*}\right)$, that

$$
\sum_{k=1}^{\infty} q\left(\xi_{k}, \xi_{k+1}\right)<\infty
$$

We conclude that $\left\{\xi_{n}\right\}$ is a left $K$-Cauchy sequence. On account of the left K-completeness, there exists $\xi^{*} \in X$, such that $\xi_{n} \stackrel{q}{\longrightarrow} \xi^{*}$.

Given the function $h \xi:=H(\xi, F \xi)$, observe that the sequence $\left\{h \xi_{n}\right\}=\left\{H\left(\xi_{n}, F \xi_{n}\right)\right\}$ is decreasing and it converges to 0 . Recall that $h$ is $\tau(q)$-lower semicontinuous (as supremum of $\tau(q)$ )-lower semicontinuous functions), which yields

$$
0 \leq h \xi^{*} \leq \liminf _{n \rightarrow \infty} h \xi_{n}=0 .
$$

Hence, $h \xi^{*}=0$, i.e. $H\left(\left\{\xi^{*}\right\}, F \xi^{*}\right)=0$. This completes the proof.

Remark 4. It is clear that, if we replace the condition (2) by the dual condition

$$
H(F \eta, \eta) \leq q(\eta, \xi)-\gamma(q(\eta, \xi)),
$$

then the conclusion of Theorem 1 would be that the multi-valued function $F$ possesses an end-point. Moreover for the multi-valued function $F$ to admit a fixed point, it is enough that

$$
H^{d_{q}}(F \eta, \eta) \leq \min \{q(\xi, \eta)-\gamma(q(\xi, \eta)), q(\eta, \xi)-\gamma(q(\eta, \xi))\},
$$

where

$$
H^{d_{q}}(A, B)=\max \left\{\sup _{a \in A} d_{q}(a, B), \sup _{b \in B} d_{q}(A, b)\right\} \text { whenever } A, B, \in \mathscr{P}_{0}(X) .
$$

If let $\gamma(t)=(1-k) t$ for $k \in[0,1)$ in Theorem 1 , then we obtain the following version of Nadler's theorem in the setting of left $K$-complete quasi-pseudo metric space.

Theorem 2. Let $(X, q)$ be a left K-complete quasi-pseudo metric space and $F: X \rightarrow \mathscr{P}_{c b}(X)$ be a multi-valued mapping. If there exists $k \in[0,1)$, such that, for each $\xi \in X$, there exists $\eta \in F \xi$ satisfying

$$
H(\eta, F \eta) \leq k q(\xi, \eta)
$$

then F possesses a start-point in X.

We conclude this part of the paper with the following illustrative example: 
Example 12. Let

$$
X=\left\{\frac{1}{2^{n}}: n=0,1,2, \cdots\right\} \cup\{0\}
$$

and let

$$
q(\xi, \eta)=\left\{\begin{array}{lc}
\eta-\xi, & \text { if } \eta \geq \xi \\
2(\xi-\eta), & \text { if } \xi>\eta .
\end{array}\right.
$$

Subsequently, $(X, q)$ is a left K-complete $T_{0}$-quasi-metric space. Set $\gamma(t)=\frac{t}{2}$ for all $t \geq 0$. Let $F: X \rightarrow$ $\mathscr{P}_{c b}(X)$ be a multi-valued map defined as

$$
F \xi=\left\{\begin{array}{l}
\left\{\frac{1}{2^{n+1}}, 0\right\} \quad \text { if } \xi=\frac{1}{2^{n}}: n=0,1,2, \cdots, \\
\{0\}, \quad \text { if } \xi=0 .
\end{array}\right.
$$

We now show that $F$ satisfies condition (2).

Case 1. $\xi=0$, there exists $\eta=0 \in F \xi=F 0=\{0\}$ such that

$$
0=H(\eta, F \eta)=H(0, F 0) \leq q(0,0)-\gamma(q(0,0))=0 .
$$

Case 2. $\xi=\frac{1}{2^{n}}$, there exists $\eta=0 \in F \xi=\left\{\frac{1}{2^{n}}, 0\right\}$, such that

$$
0=H(\eta, F \eta)=H(0, F 0) \leq q\left(\frac{1}{2^{n}}, 0\right)-\gamma\left(q\left(\frac{1}{2^{n}}, 0\right)\right)
$$

The map F satisfies the assumptions of Theorem 1, so it has a start-point, which, in this case, is 0.

In the case of a single-valued mapping, Theorem 1 produces the following existence result.

Theorem 3. Let $(X, q)$ be a left K-complete quasi-pseudo metric space and $f: X \rightarrow X$ be a weakly contractive single-valued mapping. Subsequently, $f$ possesses at least one start-point in $X$, i.e., there exists $\xi^{*} \in X$, such that $q\left(\mathcal{\zeta}^{*}, f \mathcal{\zeta}^{*}\right)=0$.

We conclude the paper with a start-point result for a multi-valued mapping satisfying a stronger weakly contractive type condition. In this case, we can obtain a stability result for the start-point problem.

Definition 9. Let $(X, q)$ be $T_{0}$-quasi-metric space. A multi-valued mapping $F: X \rightarrow \mathscr{P}_{0}(X)$ is called s-weakly contractive if there exists a $(c)^{*}$-comparison function $\gamma$, such that, for each $\xi \in X$, there exists $\eta \in F \xi$ satisfying

$$
H(\eta, F v) \leq q(\xi, v)-\gamma(q(\xi, v)), \text { for every } v \in X .
$$

Notice that any s-weakly contractive multi-valued mapping is weakly contractive, but not reversely.

The following existence and stability result holds for s-weakly contractive multi-valued mappings. For the sake of simplicity, we will present the result when $\gamma(t)=(1-k) t, t \in[0, \infty)$, with some $k \in[0,1)$.

Theorem 4. Let $(X, q)$ be a left K-complete quasi-pseudo metric space and $F: X \rightarrow \mathscr{P}_{c b}(X)$ be a multi-valued mapping. Suppose that there exists $k \in[0,1)$, such that, for each $\xi \in X$, there exists $\eta \in F \xi$ satisfying

$$
H(\eta, F v) \leq k q(\xi, v), \text { for every } v \in X .
$$


Then:

(a) F possesses a start-point in X; and,

(b) the start-point problem for $F$ is Ulam-Hyers stable with respect to the end-point problem for $F$, in the sense that there exists $C>0$, such that, for any $\varepsilon>0$ and any $\rho^{*} \in X$ with $H\left(F \rho^{*}, \rho^{*}\right) \leq \varepsilon$, there exists a start-point $\xi^{*} \in X$ of $F$, such that $q\left(\xi^{*}, \rho^{*}\right) \leq C \varepsilon$.

\section{Proof.}

(a) follows by Theorem 1. Denote, by $\xi^{*} \in X$, a start-point of $F$.

(b) For any $u \in F \xi^{*}$, we can write

$$
q\left(\xi^{*}, \rho^{*}\right) \leq H\left(\xi^{*}, F \xi^{*}\right)+H\left(u, F \rho^{*}\right)+H\left(F \rho^{*}, \rho^{*}\right)=H\left(u, F \rho^{*}\right)+H\left(F \rho^{*}, \rho^{*}\right) .
$$

For $\xi^{*} \in X$, there exists $u^{*} \in F \xi^{*}$, such that $H\left(u^{*}, F \rho^{*}\right) \leq k q\left(\xi^{*}, \rho^{*}\right)$.

Thus,

$$
q\left(\xi^{*}, \rho^{*}\right) \leq \frac{1}{1-k} \varepsilon
$$

Author Contributions: Conceptualization, Y.U.G., E.K., A.P. and S.R.; investigation, Y.U.G., E.K., A.P. and S.R.; writing-review and editing, Y.U.G., E.K., A.P. and S.R. All authors have read and agreed to the published version of the manuscript.

Funding: This research received no external funding.

Acknowledgments: Y.U.G. wishes to thank the Sefako Makgatho Health Sciences University (SU), in South Africa, which accepted him as a visitor in November 2020 and provided funding for his stay.

Conflicts of Interest: The authors declare no conflict of interest.

\section{References}

1. Gaba, Y.U. Start-points and $(\alpha, \gamma)$-contractions in quasi-pseudo metric spaces. J. Math. 2014, $2014,709253$. [CrossRef]

2. Agarwal, R.P.; Karapinar, E.; O’Regan, D.; Roldán-López-de-Hierro, A.F. Fixed Point Theory in Metric Type Spaces; Springer International Publishing Switzerland: Cham, Switzerland, 2015.

3. Ćirić, L. Some Recent Results in Metrical Fixed Point Theory; University of Belgrade: Beograd, Serbia, 2003.

4. Kirk, W.; Shahzad, N. Fixed Point Theory in Distance Spaces; Springer International Publishing Switzerland: Berlin/Heidelberg, Germany, 2014.

5. Todorčević, V. Harmonic Quasiconformal Mappings and Hyperbolic Type Metrics; Springer: Cham, Switzerland, 2019.

6. Gaba, Y.U. Advances in start-point theory for quasi-pseudo metric spaces. Bull. Allahabad Math. Soc. 2015, 30, 119-146.

7. Gaba, Y.U. New Results in the start-point theory for quasi-pseudo metric spaces. J. Oper. 2014, $2014,741818$.

8. Alegre, C.; Marin, J. Modified $w$-distances on quasi-metric spaces and a fixed point theorem on complete quasi-metric spaces. Topol. Appl. 2016, 203, 32-41. [CrossRef]

9. Alegre, C.; Marin, J. A Caristi fixed point theorem for complete quasi-metric spaces by using mw-distances. Fixed Point Theory 2018, 19, 25-31. [CrossRef]

10. Künzi, H.-P. An introduction to quasi-uniform spaces. Contemp. Math. 2009, 486, 239-304.

11. Marín, J.; Romaguera, S.; Tirado, P. Generalized contractive set-valued maps on complete preordered quasi-metric spaces. J. Funct. Spaces Appl. 2013, 2013, 269246. [CrossRef]

12. Marín, J.; Romaguera, S.; Tirado, P. Q-functions on quasimetric spaces and fixed points for multivalued maps. Fixed Point Theory Appl. 2011, 2011, 603861. [CrossRef]

13. Romaguera, S.; Tirado, P. A characterization of Smyth complete quasi-metric spaces via Caristi's fixed point theorem. Fixed Point Theory Appl. 2015, 2015, 183. [CrossRef] 
14. Mlaiki, N.; Kukić, K.; Gardaševixcx-Filipovixcx, M.; Aydi, H. On almost $b$-metric spaces related fixed points results. Axioms 2019, 8, 70. [CrossRef]

15. Mustafa, Z.; Huang, H.; Radenović, S. Some remarks on the paper "Some fixed point generalizations are not real generalizations". J. Adv. Math. Stud. 2016, 9, 110-116.

16. Romaguera, S.; Tirado, P. A characterization of quasi-metric completeness in terms of $\alpha$ - $\psi$-contractive mappings having fixed points. Mathematics 2020, 8, 16. [CrossRef]

17. Petruşel, A.; Petruşel, G. Multivalued Picard operators. J. Nonlinear Conxex Anal. 2012, 13, 157-171.

18. Petruşel, A.; Rus, I.A.; Şerban, M.A. Basic problems of the metric fixed point theory and the relevance of a metric fixed point theorem for multivalued operators. J. Nonlinear Convex Anal. 2014, 15, 493-513.

19. Petruşel, A.; Petruşel, G. Some variants of the contraction principle for multi-valued operators, generalizations and applications. J. Nonlinear Convex Anal. 2019, 20, 2187-2203.

20. Goubault-Larrecq, J. Non-Hausdorff Topology and Domain Theory: Selected Topics in Point-Set Topology; Cambridge University Press: Cambridge, UK, 2013; Volume 22.

21. Felhi, A.; Sahmim, S.; Aydi, H. Ulam-Hyers stability and well-posedness of fixed point problems for $\alpha$ - $\lambda$-contractions on quasi $b$-metric spaces. Fixed Point Theory Appl. 2016, 2016, 1. [CrossRef]

22. Reilly, I.L.; Subrahmanyam, P.V.; Vamanamurthy, M.K. Cauchy sequences in quasi-pseudo-metric spaces. Monatshefte Math. 1982, 93, 127-140. [CrossRef]

23. Alber, Y.I.; Guerre-Delabriere, S. Principles of weakly contractive maps in Hilbert spaces. In New Results in Operator Theory. Operator Theory: Advances and Applications; Gohberg, I., Lyubich, Y., Eds.; Birkhäuser: Basel, Switzerland, 1997; Volume 98, pp. 7-22._2. [CrossRef]

24. Aydi, H.; Karapinar, E.; Shatanawi, W. Coupled fixed point results for weakly contractive condition in ordered partial metric spaces. Comput. Math. Appl. 2011, 62, 4449-4460. [CrossRef]

25. Chi, K.P.; Karapinar, E.; Thanh, T.D. On the fixed point theorems in generalized weakly contractive mappings on partial metric spaces. Bull. Iranian Math. Soc. 2013, 39, 369-381.

26. Karapinar, E. Fixed point theory for cyclic weak $\phi$-contraction. Appl. Math. Lett. 2011, 24, 822-825. [CrossRef]

27. Karapinar, E. Weak $\phi$-contraction on partial contraction. J. Comput. Anal. Appl. 2012, 14, 206-210.

28. Karapinar, E. Best proximity points of Kannan type cylic weak phi-contractions in ordered metric spaces. Analele Stiintifice Ale Univ. Ovidius Constans 2012, 20, 51-64.

29. Rhoades, B.E. Some theorems on weakly contractive maps. Nonlinear Anal. 2001, 47, 2683-2693. [CrossRef]

30. Browder, F.E. On the convergence of successive approximations for nonlinear functional equations. Nederl. Akad. Wetensch. Ser. A71 Indag. Math. 1968, 30, 27-35. [CrossRef]

31. Rus, I.A. Generalized Contractions and Applications; Cluj University Press: Cluj-Napoca, Romania, 2001.

32. Berinde, V. Generalized contractions in quasi-metric spaces. In Seminar on Fixed Point Theory; Research Seminars No. 3; Babeş-Bolyai University: Cluj-Napoca, Romania, 1993; pp. 3-9.

Publisher's Note: MDPI stays neutral with regard to jurisdictional claims in published maps and institutional affiliations.

(C) 2020 by the authors. Licensee MDPI, Basel, Switzerland. This article is an open access article distributed under the terms and conditions of the Creative Commons Attribution (CC BY) license (http://creativecommons.org/licenses/by/4.0/). 